MITSUBISHI ELECTRIC RESEARCH LABORATORIES
http://www.merl.com

\title{
Fault Location Analysis of Ungrounded Distribution System Based on Residual Voltage Distribution
}

\author{
Sun, H.; Nikovski, D.; Takano, T.; Kojima, Y.; Ohno, T.
}

TR2013-089 September 2013

\begin{abstract}
This paper proposes a new method for determining the location of single-phase-to-ground faults of ungrounded distribution systems based on residual voltage distribution. The possible faulted line segment and fault location are determined by comparing the phase angles of faulted phase voltages against a faulted phase reference angle, and the faulted phase voltages are determined as the difference between the fault caused residual voltage of all phases and the shunt caused residual voltage of un-faulted phases. The method determines the fault location solely based on the voltages and currents measured during the fault, and associated series impedance and shunt impedances of line segments. It does not require any measurements or information for the load demands and pre-fault conditions. Numerical examples are given to demonstrate the effectiveness of the proposed method.
\end{abstract}

North American Power Symposium (NAPS)

\footnotetext{
This work may not be copied or reproduced in whole or in part for any commercial purpose. Permission to copy in whole or in part without payment of fee is granted for nonprofit educational and research purposes provided that all such whole or partial copies include the following: a notice that such copying is by permission of Mitsubishi Electric Research Laboratories, Inc.; an acknowledgment of the authors and individual contributions to the work; and all applicable portions of the copyright notice. Copying, reproduction, or republishing for any other purpose shall require a license with payment of fee to Mitsubishi Electric Research Laboratories, Inc. All rights reserved.
} 



\section{Fault Location Analysis of Ungrounded Distribution System Based on Residual Voltage Distribution}

\author{
Hongbo Sun, Daniel Nikovski \\ Mitsubishi Electric Research Laboratories \\ Cambridge, MA 02139 USA
}

\author{
Tomihiro Takano, Yasuhiro Kojima, Tetsufumi Ohno \\ Mitsubishi Electric Corporation \\ Hyogo 661-8661 Japan
}

\begin{abstract}
This paper proposes a new method for determining the location of single-phase-to-ground faults of ungrounded distribution systems based on residual voltage distribution. The possible faulted line segment and fault location are determined by comparing the phase angles of faulted phase voltages against a faulted phase reference angle, and the faulted phase voltages are determined as the difference between the fault caused residual voltage of all phases and the shunt caused residual voltage of un-faulted phases. The method determines the fault location solely based on the voltages and currents measured during the fault, and associated series impedance and shunt impedances of line segments. It does not require any measurements or information for the load demands and prefault conditions. Numerical examples are given to demonstrate the effectiveness of the proposed method.
\end{abstract}

\section{INTRODUCTION}

Ungrounded distribution systems are widely used, especially at medium voltage levels. Compared with the grounded distribution systems, the ungrounded systems do not have neural wires to connect with the ground, and they are connected to ground through phase-to-ground capacitances of power lines. When a single-phase-to-ground fault occurs, the fault currents of ungrounded distribution systems are usually less than normal load currents, thus the system can continue to operate until the fault is corrected. However, as a result of the fault, the distribution systems experience over-voltages, which can cause equipment damages when the fault is not corrected in a timely manner. Thus, fault location analysis is important for the safe and stable operation of ungrounded distribution systems.

Several fault location analysis algorithms have been proposed for distribution systems, including impedance-based methods [1-2], direct circuit analysis methods [3], transient based methods [4], signal injection methods [5], and knowledge-based approaches [6]. Those methods have their own limitations when applied to real time analysis of ungrounded distribution systems. Impedance based methods and direct circuit analysis methods are more generic, and much easier for implementation than other methods, but most of existing methods are designed for grounded distribution systems. Transient based approaches may get prediction results before the fault currents get into steady states, but usually require much higher sampling rate, and might not be cost effective. Signal injection methods use additional frequency signals and measures to locate the fault, and are only suitable for off-line applications. Knowledge based methods may get more accurate results if pre-training sets are closely matching with new fault events, but requires more computation efforts that other ones.

This paper proposes a new method to determine the location of a single-phase-to-ground fault in an ungrounded distribution system based on the fundamental frequency measurements collected from the feeder breakers at the substation and the intelligent switches along the feeders. The proposed method firstly determines the faulted phase, faulted feeder and faulted feeder section based on the voltage and current measurements collected during the fault, and then determines the possible faulted line segment and fault location by detailed analysis of residual voltage distribution in the faulted feeder section. The fault location analysis is solely based on the voltage and current measurements during the fault, and associated series impedance and shunt admittances of line segments in the feeder sections. It does not need any information or measurements for the load demands of the system or the pre-fault conditions in the system.

The method is proposed based on an observation that a faulted line segment can be determined by comparing the phase angle of the phase-to-ground voltage of the faulted phase against a reference angle of the faulted phase if assuming that the fault current flows across the line segment instead of entering into ground through a location within the line segment. If the value of the reference angle of the faulted phase is between the phase angles of the voltages on the bus terminals forming the line segment, then this line segment is faulty. However, in order to determine the voltage on the buses, the loads of the buses should be determined, which can be a difficult task. Thus, it is desired to avoid the determination of the loads. In the proposed method, the voltage on the faulted phase is approximated with a difference between the fault caused residual voltage of all phases and the shunt caused residual voltage of un-faulted phases. The shunt caused residual voltages are used to describe the residual voltage distribution of the feeder section under an un-faulted condition with given measured voltages at the boundaries of 
the feeder section, and the fault caused residual voltages are used to describe the residual voltage distribution under a faulted condition that occurred downstream to the location under consideration. The faulted line segment is identified when the faulted phase reference angle is between the phase angles of voltages on the faulted phase determined at two terminal buses of a line segment. The fault point is determined by finding a location along the faulted line that the phase angle of voltage on the faulted phase is in phase with the faulted phase reference angle. Numerical examples are given to demonstrate the effectiveness of the proposed method.

\section{PROPOSED METHOD}

\section{A. Ungrounded Distribution System and Measurements}

Fig. 1 shows an example of ungrounded distribution system with a single-phase-to-ground fault. It includes a distribution substation in which a three-phase transformer with ungrounded winding connections is connected with downstream feeders, and each feeder has one feeder breaker and several intelligent switches.

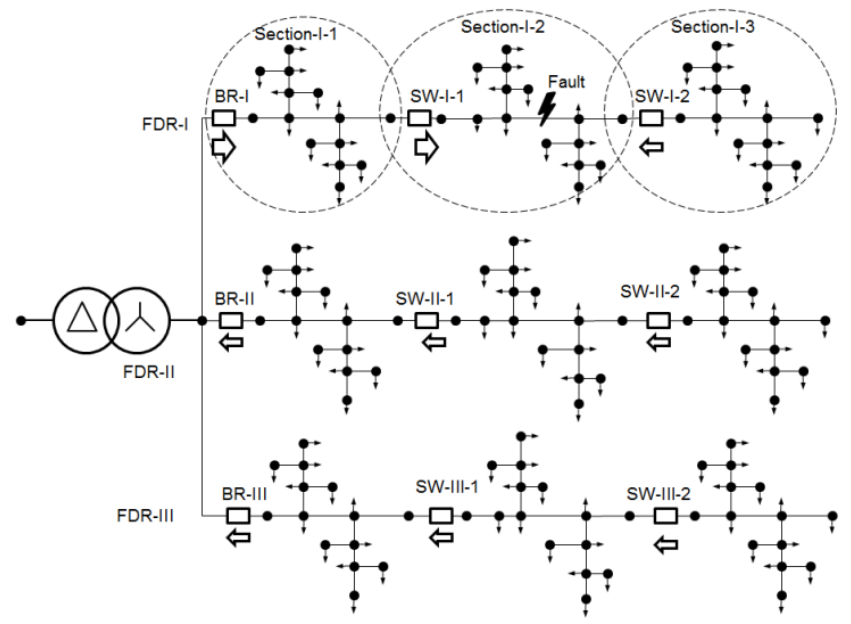

Figure 1. Example of an ungropunded distribution system

According to the location of switches along the feeder, a feeder can be partitioned into several sections. Each feeder section has one feeder breaker or intelligent switch at its root as an importing measuring device, and may have one or multiple intelligent switches at its downstream boundaries as exporting measuring devices. The feeder section is defined as all buses and devices between the importing and exporting measuring devices. In Fig. 1, the system has three feeders, each feeder has one breaker and two switches, and each feeder includes three feeder sections.

The buses of a feeder section can be grouped into several layers according to the number of devices connected between each bus and the importing measuring device. The higher the layer number is, and the farther the buses of the layer are from the importing device.

The measurements collected from feeder breakers and intelligent switches include the currents flowing through the device downstream on phase $a, b$ and $c, I_{p s, a}, I_{p s, b}$ and $I_{p s, c}$, and the phase-to-ground voltages on phase $a, b$ and $c, V_{p, a}, V_{p, b}$ and $V_{p, c}$, where bus $p$ and $s$ are the terminal buses of the breaker or switch, and bus $p$ is upstream to bus $s$.

Based on the measured phase-to-ground voltages and phase currents, the residual voltage and residual current for a device between bus $p$ and bus $s$ can be determined according to:

$$
\begin{aligned}
& v_{p}^{r e s}=V_{p, a}+V_{p, b}+V_{p, c} \\
& i_{p s}^{r e s}=I_{p s, a}+I_{p s, b}+I_{p s, c}
\end{aligned}
$$

where, $v_{p}^{r e s}$ is the residual voltage at bus $p, i_{p s}^{\text {res }}$ is the residual current flowing from bus $p$ to bus $s$ and measured at bus $p$.

\section{B. Determination of faulted phase, faulted feeder, and faulted feeder section}

The faulted phase is determined using the phase-to-ground voltages measured at the secondary side of the substation transformer, i.e. the upstream bus of feeder breakers.

When a bolted single-phase-to-ground fault occurs at a feeder, the phase-to-ground voltage of the faulted phase on the faulted feeder, and adjacent feeders that connected to the same substation transformer with the faulted feeder instantaneously drops to a small value close to zero. On the other hand, due to the ungrounded winding connection of the transformer, the substation maintains the phase-to-phase voltage close to unchanged, and then the phase-to-ground voltages of the other two un-faulted phase instantaneously rise to values close to 1.73 times of its normal operation ones.

The phase $x$ is determined as faulted phase, if the following conditions are met:

$$
\begin{array}{cl}
\left|V_{s u b, x}\right| \leq V & x \in\{a, b, c\} \\
\left|V_{s u b, y}\right| \geq \bar{V} & y \in\{a, b, c\}, y \neq x
\end{array}
$$

where, $V_{s u b, x}$ and $V_{s u b, y}$ are the phase-to-ground voltage measured at the secondary side of the substation transformer on the phase $x$ and $y$ respectively, and $\underline{V}$ and $\bar{V}$ are the lower and upper thresholds of voltage magnitude used for abnormal voltage determination. For examples, the lower and upper thresholds can be set as 0.30 , and 1.40 per unit respectively.

The faulted feeder and faulted feeder section are determined based on the phase angle difference between residual voltage and residual currents at the measuring devices along the feeders.

If ignoring the asymmetry of power lines, the residual currents of ungrounded distribution systems are majorly contributed by the phase-to-ground capacities of un-faulted phases of the faulted feeder and un-faulted feeders. When a bolted single-phase-to-ground fault occurs at a location within a feeder, the residual voltage at the location will rise to a value close to 3 times of normal phase-to-ground voltage of faulted phase. The direction of residual currents at a location downstream to the faulted location is flowing toward the substation, so the residual voltage is leading residual current around 90 degree. On the other hand, the direction of residual current at a location upstream to the fault is flowing toward the faulted location and away from the substation, so the residual voltage is lagging the residual current around 90 degree. 
A feeder is determined as a faulted one if the phase angle difference between the residual voltage and residual current measured at the feeder breaker are close to 90 degree as described in Eq. (5):

$$
\left|\angle v_{f d r}^{r e s}-\angle i_{f d r}^{r e s}-90^{\circ}\right|<\Delta \bar{\theta}
$$

where, $\angle v_{f d r}^{r e s}$ is the phase angle of residual voltage measured at the upstream terminal bus of the feeder breaker, $\angle i_{f d r}^{r e s}$ is the phase angle of residual current flowing into the feeder breaker through its upstream terminal bus, $\Delta \bar{\theta}$ is a pre-determined upper threshold of angle difference according to the ratio of shunt sucesptance components over total shunt admittances of typical conductors used in the distribution systems. For example, $\Delta \bar{\theta}$ is set to be 20 degree.

The faulted feeder section is determined by checking the angle difference between the residual voltage and residual current of the measuring devices at the boundaries of the feeder section. A feeder section is determined to be a faulted one when the angle difference between residual voltage $v_{i m}^{\text {res }}$ and residual current $i_{\text {im }}^{r e s}$ at its importing measuring device $i m$ is close to 90 degree as described in Eq. (6):

$$
\left|\angle v_{i m}^{r e s}-\angle i_{i m}^{r e s}-90^{\circ}\right|<\Delta \bar{\theta}
$$

and the angle difference between residual voltage $v_{e x}^{r e s}$ and residual current $i_{e x}^{r e s}$ at one of its exporting measuring device $e x$ is close to -90 degree as described in Eq. (7):

$$
\left|\angle v_{e x}^{r e s}-\angle i_{e x}^{r e s}+90^{\circ}\right|<\Delta \bar{\theta}
$$

If the magnitude of residual current at the importing measuring device is close to be zero, only Eq. (7) is used to determine whether there is a fault within the section based on the measurements at the exporting measuring devices. If a feeder section does not have any exporting measuring device, only Eq. (6) is used to determine whether it is a faulted section using the measurements at the importing device.

\section{Determining of shunt caused residual voltage distribution}

The shunt caused residual voltages are used to describe the residual voltage distribution of a feeder section with given measured voltages at the boundaries of the section if no fault occurs in the section.

The shunt caused residual voltage of all phases at bus $p$, $\hat{v}_{p}^{r e s}$ can be determined based on the estimated phase-toground voltages at bus $p$, according to:

$$
\hat{v}_{p}^{r e s}=\sum_{x \in\{a, b, c\}} \hat{V}_{p, x}
$$

where, $\widehat{V}_{p, x}$ is the estimated phase-to-ground voltages of phase $x$ at bus $p$. If phase $x$ is the faulted phase, the shunt caused residual voltage of un-faulted phases at bus $p$ is determined as:

$$
\hat{v}_{p, \text { unfaulted }}^{\text {res }}=\sum_{y \neq x, y \in\{a, b, c\}} \widehat{V}_{p, y}
$$

where, $y$ is the un-faulted phase.

For a feeder section, the estimated voltage distribution within the section can be determined based on the phase-toground voltage measurements at the importing and exporting measuring devices of the section and its topology connectivity.

The voltages at buses connected to the measuring devices are directly set as the measured values.
For any bus residing in the connectivity path between each pair of the importing measuring device and one of the exporting measuring devices, its estimated phase-to-ground voltages are determined based its distances to two measuring devices and measured voltages at two measuring devices. If there are multiple exporting measuring devices existing, and common buses between different paths, the voltages of those common buses are set as the average of estimated voltages for all paths.

The estimated phase-to-ground voltage of bus $p$ is determined according to:

$$
\widehat{V}_{p}=\frac{1}{m} \sum_{i=1}^{m}\left(\frac{d_{p-e x_{i}}}{d_{i m-p}+d_{p-e x_{i}}} V_{i m}+\frac{d_{i m-p}}{d_{i m-p}+d_{p-e x_{i}}} V_{e x_{i}}\right)
$$

where, $\widehat{V}_{p}$ is the vector of estimated phase-to-ground voltages of bus $p, m$ is the total number of paths that pass through bus $p, V_{i m}$ and $V_{e x_{i}}$ are the phase-to-ground voltages measured at the importing measuring device $i m$ and $i$-th exporting measuring device $e x_{i}, d_{i m-p}$ and $d_{p-e x_{i}}$ are the sum of length of line segments residing at the path between the importing device $i m$ and bus $p$, and between bus $p$ and the $i$-th exporting measuring device $e x_{i}$ respectively.

For any bus not residing on any paths between the measuring devices, but fed from one of buses in the paths, its estimated voltage is set as the estimated voltage of the feeding bus on the paths. If a feeder section does not have any exporting measuring device, the estimated voltages of buses within the section are set as the measured voltages at the importing measuring device.

\section{Determining of shunt caused residual current distribution}

If there is no fault in the feeder section, the residual currents on each line segment are solely contributed by the shunt currents of line segments downstream to the importing measuring device of the feeder section. The shunt currents can be determined based on the estimated voltages of terminal buses of associated line segments and shunt admittance of those segments, when the asymmetry of power lines is ignored. This type of residual currents is called shunt caused residual currents.

The contributions of residual currents from each phase are modeled and solved separately. For example, the residual current entering a line segment between bus $p$ and bus $s$ through bus $p, i_{p s}^{\text {res }}$ can be determined according to:

$$
i_{p s}^{r e s}=\sum_{x \in\{a, b, c\}} I_{p s, x}^{r e s}
$$

where, $I_{p s, x}^{r e s}$ is the residual current component of line segment between bus $p$ and bus $s$ contributed from phase $x$. The residual currents contributed from all phases can be described using a vector $I_{p s}^{r e s}$, and $I_{p s}^{r e s}=\left[\begin{array}{lll}I_{p s, a}^{r e s} & I_{p s, b}^{r e s} & I_{p s, c}^{r e s}\end{array}\right]^{T}$.

A backward sweep procedure is used to determine the shunt caused residual current distribution in the feeder section. The procedure starts at the line segments connected with the last layer of the feeder section, then moves backward to next upstream layer, and ends at the line segments connected to the first layer of the section.

For any device between an upstream bus $p$ and a downstream bus $s$, two residual currents need to be determined, one is the ones entering the device through the 
upstream bus $p$, and the other is the ones leaving the device through the downstream bus $s$. Taken a line segment as example, the residual currents leaving through the downstream bus of the line segment is determined first according to the residual currents on the line segments downstream. Then the residual currents entering the line segment through the upstream bus is determined based on the residual currents leaving through the downstream bus, and the shunt currents contributed from the shunt admittances of the line segment.

Fig. 2 shows an example of a line segment between an upstream bus $p$ and a downstream bus $s$. The line segment is modeled by a series phase impedance matrix $Z_{p s}^{s e}$, and a shunt admittance matrix $Y_{p s}^{s h}$ partitioned into two terminal buses. The phase-to-ground voltages at bus $p$ and bus $s$ are represented by the vectors $V_{p}$ and $V_{s}$, and the residual voltages at bus $p$ and bus $s$ are represented by the variables $v_{p}^{r e s}$ and $v_{s}^{r e s}$. The phase residual currents flowing on the line segments are represented by the vectors $I_{p s}^{r e s}$ and $I_{p s^{\prime}}^{r e s}, I_{p s}^{r e s}$ is the vector of residual currents entering into the line segment through bus $p$, and $I_{p s^{\prime}}^{r e s}$ is the vector of residual currents leaving from the line segment through bus $s$.

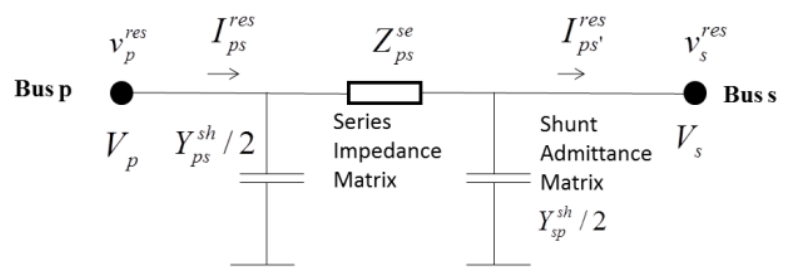

Figure 2. Example of a line segment

For a line segment between an upstream bus $p$ and a downstream bus $s$, the vector of shunt caused residual currents leaving the segment through the downstream bus $s, \hat{I}_{p s^{\prime}}^{r e s}$ is determined as:

$$
\hat{I}_{p s^{\prime}}^{r e s}=\sum_{t \in D D_{s}} \hat{I}_{s t}^{r e s}
$$

where, $D D_{s}$ is the set of downstream buses that directly connected with bus $s$ through a line segment or switch. $\hat{I}_{s t}^{r e s}$ is the vector of residual currents entering a line segment or switch between bus $s$ and bus $t$ through bus $s$. The vector of shunt caused residual currents entering the segment between bus $p$ and $s$ through the upstream bus $p, \hat{I}_{p s}^{\text {res }}$ is determined as:

$$
\hat{I}_{p s}^{r e s}=\hat{I}_{p s^{\prime}}^{r e s}+\frac{1}{2} Y_{p s}^{s h}\left(\hat{V}_{p}+\hat{V}_{s}\right)
$$

where, $\widehat{V}_{s} i$ s the vector of estimated phase-to-ground voltages of bus $s$. Eq. (12) and (13) can be also applied to a switch between bus $p$ and bus $s$, by setting the shunt admittance matrix as zero.

If the downstream bus $s$ of the line segment between bus $p$ and $s$ is an upstream bus of an exporting measuring device, the residual currents leaving through bus $s$ are determined based on the measurements at the exporting device, and shunt currents downstream to the exporting device according to:

$$
\begin{aligned}
& \hat{I}_{p s^{\prime}, x}^{r e s}=i_{e x}^{r e s}-\sum_{y \neq x, y \in\{a, b, c\}} \hat{I}_{p s^{\prime}, y}^{r e s} \\
& \hat{I}_{p s^{\prime}, y}^{r e s}=\sum_{m n \in D N_{e x}} \frac{1}{2} Y_{m n, y}^{s h}\left(\hat{V}_{m}+\widehat{V}_{n}\right)
\end{aligned}
$$

where, $\hat{I}_{p s^{\prime}, x}^{r e s}$ and $\hat{I}_{p s^{\prime}, y}^{r e s}$ are the shunt caused residual currents leaving the line segment between bus $p$ and bus $s$ through bus $s$ on the faulted phase $x$, and one of un-faulted phase $y, i_{e x}^{r e s}$ is the measured residual current at the exporting measuring device, $D N_{e x}$ is the set of all line segments downstream to the exporting measuring device, $Y_{m n, y}^{s h}$ is the vector of shunt admittance elements of line segment between bus $m$ and bus $n$ at the row corresponding to the un-faulted phase $y, \widehat{V}_{m}$ and $\widehat{V}_{n}$ are the vectors of estimated voltages of bus $m$ and bus $n$.

\section{E. Determining of fault caused residual current distribution}

For a line segment, if a fault is occurring downstream to its downstream terminal bus, its residual current of the faulted phase is mainly contributed from the shunt currents on the unfaulted phases of the faulted feeder and adjacent feeders connected to the same substation transformer with the faulted feeder. This type of residual currents is called fault caused residual currents. The fault caused residual currents are determined for each line segment in the faulted section, and used to determine a corresponding fault caused voltage for determining the possible faulted line segments.

The distribution of fault caused residual currents are determined based on the residual current measurements at the root of the faulted section, the estimated phase-to-ground voltages and shunt admittances of line segments through a forward sweep procedure. The fault current of a line segment is calculated by sequentially assuming a fault located downstream to its downstream terminal bus, starting from the devices connected to the root of the feeder section and towards the ends of the feeder section.

For any line segment connected an upstream bus $p$ to a downstream bus $s$, the fault caused residual current entering the segment through the upstream bus $p$ is determined according to:

$$
\tilde{I}_{p s}^{r e s}=\tilde{I}_{d p^{\prime}}^{r e s}-\sum_{t \in D D_{p}, t \neq s} \hat{I}_{p t}^{r e s}
$$

where, $\tilde{I}_{p s}^{r e s}$ is the vector of fault caused residual currents entering the segment through bus $p, \tilde{I}_{d p^{\prime}}^{r e s}$ is the vector of fault caused residual currents leaving an upstream line segment between bus $d$ and bus $p$ through bus $p, D D_{p}$ is the set of buses downstream to bus $p$ and having direct connection with bus $p$ through a line segment or switch, $\hat{I}_{p t}^{r e s}$ is the vector of shunt caused residual current entering the device between bus $p$ and bus $t$ through bus $p$. The fault caused residual current leaving the line segment through the downstream bus is determined as:

$$
\tilde{I}_{p s^{\prime}}^{r e s}=\tilde{I}_{p s}^{r e s}-\frac{1}{2} Y_{p s}^{s h}\left(\widehat{V}_{p}+\widehat{V}_{s}\right)
$$

where, $\tilde{I}_{p s^{\prime}}^{r e s}$ is the vector of fault caused residual currents leaving the line segment through bus $s$. Eq. (16) and (17) can also be applied to a switch between bus $p$ and bus $s$, by setting the shunt admittance matrix $Y_{p s}^{s h}$ as zero.

If the upstream bus of a device between bus $p$ and $s$ is an upstream bus of an importing measuring device, the residual currents entering the device through the upstream bus are determined based on the measurements at the importing device, and shunt currents downstream to the importing device according to: 


$$
\begin{gathered}
\tilde{I}_{p s, x}^{r e s}=i_{i m}^{r e s}-\sum_{y \neq x, y \in\{a, b, c\}} \tilde{I}_{p s, y}^{r e s} \\
\tilde{I}_{p s, y}^{r e s}=\sum_{m n \in D N_{i m}} \frac{1}{2} Y_{m n, y}^{s h}\left(\widehat{V}_{m}+\widehat{V}_{n}\right)
\end{gathered}
$$

where, $\tilde{I}_{p s, x}^{r e s}$ and $\tilde{I}_{p s, y}^{r e s}$ are the residual currents entering the device between bus $p$ and bus $s$ through bus $p$ on the faulted phase $x$, and one of un-faulted phase $y, i_{i m}^{r e s}$ is the measured residual current at the importing measuring device $\mathrm{im}$, $D N_{i m}$ is the set of all line segments downstream to the importing measuring device im.

\section{F. Determining of fault caused residual voltage distribution}

The fault caused residual voltages of terminal buses are determined based on the residual voltages and residual currents measured at the root of the faulted section, fault caused residual currents, and line series impedances and shunt admittances of line segments.

Similar as the procedure used for fault caused residual current determination, a forward sweep procedure is used to determine the fault caused residual voltages for each line segment starting from the root of the feeder section towards to the end of the feeder section. The forward sweep procedure is starting from the buses of importing measuring device at the root of the feeder section, and the fault caused voltages at those buses are set to be the same as the corresponding measured residual voltages.

For any line segment between an upstream bus $p$ and a downstream bus $s$, the fault caused residual voltage at the downstream bus $s$ is determined based on the one at the upstream bus and fault caused residual currents entering the line segment through the upstream bus according to:

$$
\tilde{v}_{s}^{r e s}=\tilde{v}_{p}^{r e s}-T^{T} Z_{p s}^{s e}\left(\tilde{I}_{p s}^{r e s}-\frac{1}{2} Y_{p s}^{s h} \widehat{V}_{p}\right)
$$

where, $\tilde{v}_{s}^{\text {res }}$ and $\tilde{v}_{p}^{\text {res }}$ are the fault caused residual voltages at bus $s$ and bus $p, T$ is a vector of unity defined as $T=$ $\left[\begin{array}{lll}1 & 1 & 1\end{array}\right]^{T}$. For a switch between bus $p$ and bus $s$, the fault caused residual voltage at the downstream bus $s$ is set as the same as one of the upstream bus $p$.

\section{G. Determination of faulted line segments}

The faulted cause residual voltage for each bus is contributed from both the faulted phase and the un-faulted phases. The contribution from the faulted phase, i.e. the voltage at the faulted phase can be determined if the contributions of un-faulted phases to the faulted caused residual voltages are known. In this paper, the shunt caused residual voltages of un-faulted phases are used to approximate the faulted caused residual voltage of un-faulted phases. Thus, the faulted phase voltages on the terminal buses of a line segment between bus $p$ and bus $s$ can be determined according to:

$$
\begin{gathered}
\tilde{V}_{p, x}=\tilde{v}_{p}^{\text {res }}-\hat{v}_{p, \text { unfaulted }}^{\text {res }} \\
\tilde{V}_{s, x}=\tilde{v}_{s}^{\text {res }}-\hat{v}_{s, \text { unfaulted }}^{\text {res }}
\end{gathered}
$$

where, $\tilde{V}_{p, x}$ and $\tilde{V}_{s, x}$ are the faulted phase voltages at bus $p$, and bus $s, \hat{v}_{p, \text { unfaulted }}^{\text {res }}$ and $\hat{v}_{s, \text { unfaulted }}^{\text {res }}$ are the shunt caused residual voltages of un-faulted phases at bus $p$ and bus $s$ respectively.

The possible faulted line segments are determined by comparing the phase angle changes for the faulted phase voltages between two terminal buses of each line segment. As shown in Fig. 3, if the angles at two terminal buses are located at different sides of a reference axis defined by a reference angle according to the faulted phase, the line segment is determined to be a possible faulted one. In Fig. 3, $p$ and $s$ are the terminal buses of the line segment, and $\theta_{x}^{r e f}$ is the reference angle determined by the faulted phase.

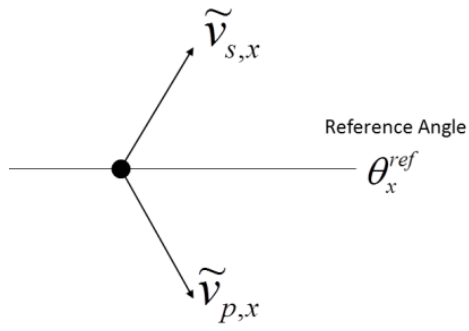

Figure 3. Terminal Residual Volatge Phasors

A line segment is determined to be a faulted one if the following condition is met:

$$
\sin \left(\angle \tilde{V}_{p, x}-\theta_{x}^{r e f}\right) \sin \left(\angle \tilde{V}_{s, x}-\theta_{x}^{r e f}\right)<0
$$

where, $\angle \tilde{V}_{p, x}$ and $\angle \tilde{V}_{s, x}$ are the phase angles of the fault phase voltages at bus $p$, and bus $s$ respectively.

$\theta_{x}^{r e f}$ is set as the phase angle of internal voltage of equivalent infinite source for transmission systems fed to the substation. For example, the reference angle can be set as 0 , 120 or 120 degree if the faulted phase is phase $a$, phase $b$, or phase $c$ respectively.

\section{H. Determination of fault location on the faulted line segment}

Knowing the possible faulted line segments, the possible fault location along each line segment can be determined according the phase angle changes between the fault and shunt caused residual voltages.

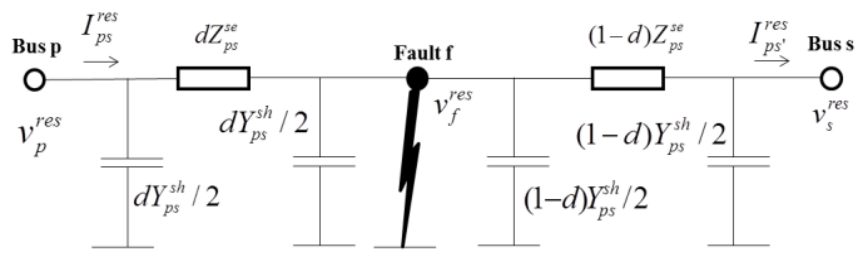

Figure 4. Model of a line segment with a fault

Assumed a single-phase-to-ground fault occurs on a line segment between an upstream bus $p$ and a downstream bus $s$, and the fault is located at point $f$ as shown in Fig. 4. The line segment can be divided into two sub-segments according to the location of the fault, one is between bus $p$ and the location of fault $f$, and the other is between location of the fault $f$ and bus $s$. Assumed $d$ is the ratio of distance between the fault location $f$ and the upstream bus $p$ over total length of the line segment between bus $p$ and bus $s$, the sub-segment between bus $p$ and fault location $f$ can be modeled with a series impedance $d Z_{p s}^{s e}$, and a shunt admittance $d Y_{p s}^{s h}$ split into two terminal buses $p$, and $f$, and the sub-segment between fault location $f$ and bus $s$ can be modeled with a series impedance 
$(1-d) Z_{p s}^{s e}$, and a shunt admittance $(1-d) Y_{p s}^{s h}$ split into two terminal buses, $f$ and $s$.

Based on the series impedance and shunt impedance model for the sub-segment between bus $p$ and fault location $f$, the fault caused residual voltage at location $f$ can be calculated as:

$$
\tilde{v}_{f}^{r e s}=\tilde{v}_{p}^{r e s}-d T^{T} Z_{p s}^{s e}\left(\tilde{I}_{p s}^{r e s}-\frac{d}{2} Y_{p s}^{s h} \widehat{V}_{p}\right)
$$

where, $\tilde{v}_{f}^{r e s}$ is the fault caused residual voltage at the location $f$. The shunt caused residual voltage of un-faulted phases at location $f$ can be calculated as:

$$
\hat{v}_{f, \text { unfaulted }}^{\text {res }}=(1-d) \hat{v}_{p, \text { unfaulted }}^{\text {res }}+d \hat{v}_{s, \text { un }}^{\text {res }}
$$
where, $\hat{v}_{f, \text { res }}^{\text {res aulted }}$ is the shunt caused residual voltage of unfaulted phases at fault location $f$.

Accordingily, the voltage of the faulted phase at the location $f, \tilde{V}_{f, x}$ can be determined as:

$$
\tilde{V}_{f, x}=\tilde{v}_{f}^{\text {res }}-\hat{v}_{f, \text { unfaulted }}^{\text {res }}
$$

The angle of the faulted phase voltage is comparing with the reference angle corresponding to the fault phase. If they are close enough, the fault is located at the location $f$.

The location $f$ is determined as the faulted point if the following condition is met:

$$
\left|\sin \left(\angle \tilde{V}_{f, x}-\theta_{x}^{r e f}\right)\right|<\varepsilon
$$

where, $\varepsilon$ is a small threshold, such as 0.00001 .

\section{NUMERICAL EXAMPLES}

The proposed method has been tested with several sample ungrounded systems, and satisfactory results are obtained.

Fig. 5 gives an example of sample distribution systems. The system includes 1 three-phase transformer, and 3 feeders. Each feeder has 1 feeder breaker, 2 intelligent switches, 18 three-phase line segments, and 22 three-phase buses. Each line segment is 1.25 miles long, and the maximum length of a single feeder is 15 miles. Both the feeder breakers and intelligent switches are equipped with measuring units for voltages and currents.

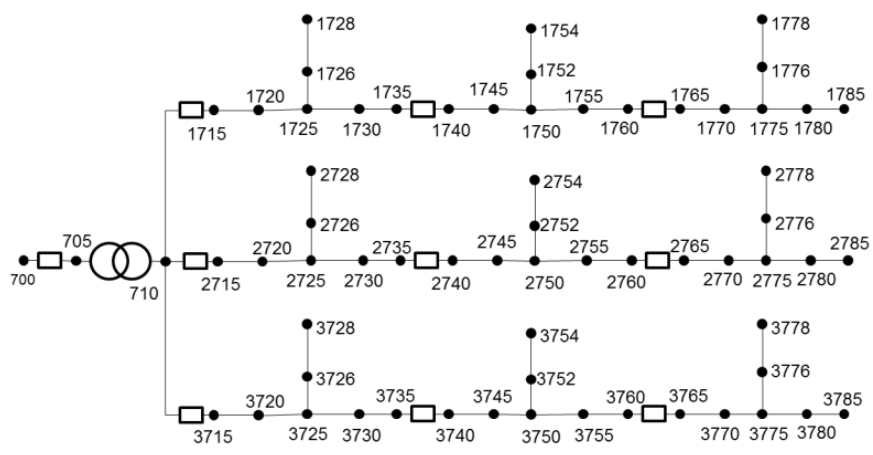

Figure 5. A sample ungrounded distribution system

Table I gives the test results on six different fault cases, and each case represents a bolted single-phase-to-ground fault at a specific location. As shown in the table, the maximum estimation error of the proposed method is 0.57675 miles. Considering the feeder length is 15 miles long, the maximum estimation error is $3.85 \%$ of feeder length.

TABLE I. TEST RESULTS ON A SAMPLE SYSTEM

\begin{tabular}{|c|c|c|}
\hline Fault Location & $\begin{array}{c}\text { Estimation Error } \\
\text { (miles) }\end{array}$ & $\begin{array}{c}\text { Estimation Error } \\
(\mathbf{\%})\end{array}$ \\
\hline 2720 & 0.57675 & 3.845 \\
\hline 2726 & 0.51350 & 3.423 \\
\hline 2745 & 0.04637 & 0.309 \\
\hline 2750 & 0.09975 & 0.665 \\
\hline 2775 & 0.00858 & 0.057 \\
\hline 2780 & 0.11125 & 0.742 \\
\hline
\end{tabular}

\section{CONCLUSIONS}

This paper has proposed a new method for locating the single-phase-to-ground faults of ungrounded distribution systems using the during-fault measurements at the feeder breakers and intelligent switches along the feeders. It does not need any information or measurements regarding the load demands of the system or the pre-fault conditions in the system.

The fault location analysis is based on comparing the phase angle of the faulted phase voltage against a faulted phase reference angle. The faulted phase voltage is determined as the difference between fault caused residual voltages of all phases and shunt caused residual voltages of un-faulted phases. The faulted line segment is identified when the faulted phase reference angle is between the phase angles of the faulted phase voltages determined at two terminal buses of the line segment. The faulted point is determined by finding a location along the faulted line segment that the phase angle of faulted phase voltage is in phase with the faulted phase reference angle.

The preliminary test results have proven the effectiveness of the proposed method.

\section{REFERENCES}

[1] "IEEE Guide for Determining Fault Location on AC Transmission and Distribution Lines", IEEE Std C37.114, pp. 1-36, 2004.

[2] R. H Salim, M. Resener, A. D. Filomena, K. Rezende Caino de Oliveira, and A. S. Bretas, "Extended fault-location formulation for power distribution systems. IEEE Transactions on Power Delivery, vol. 24, no. 2, pp. 508-516, 2009.

[3] M. S. Choi, S. J. Lee, D. S Lee, and B. G Jin, "A New Fault Location Algorithm Using Direct Circuit Analysis for Distribution Systems", IEEE Transactions on Power Delivery, vol. 19, no. 1, pp. 35-41, 2004.

[4] M. Lehtonen, "Method for distance estimation of single-phase-toground faults in electrical distribution networks with an isolated or compensated neutral", European Transactions on Electrical Power, vol. 5, no.3, pp. 193-198, 2007.

[5] T. Baldwin, F. Renovich, Jr., L. F. Saunders, and D. Lubkeman, "Fault locating in ungrounded and high-resistance grounded systems," IEEE Transactions on Industry Applications, vol. 37, pp. 1152-1159, 2001.

[6] J. Mora-Florez, V. Barrera-Nuez, and G. Carrillo-Caicedo, "Fault location in power distribution Systems using a learning algorithm for multivariable data analysis", IEEE Transactions on Power Delivery, vol. 22, no. 3, pp. 1715-1721, 2007. 